\title{
Dissociated Neurons Regenerate into Sciatic But Not Optic Nerve Explants in Culture Irrespective of Neurotrophic Factors ${ }^{1}$
}

\author{
M. E. SCHWAB ${ }^{2}$ AND H. THOENEN
}

Max Planck Institute for Psychiatry, Department of Neurochemistry, 8033 Martinsried, Federal Republic of Germany

\begin{abstract}
Explants of adult or 10-day-old rat sciatic and optic nerves were implanted as "bridges" through a silicon grease seal in a three-compartment chamber culture system, leading from a narrow center chamber to two adjacent side chambers. Dissociated newborn rat sympathetic or sensory neurons were plated into the center chamber and grown in the presence of optimal concentrations of nerve growth factor (NGF). By light microscopy, nerve fibers were seen to grow out of the sciatic nerve explants in the side chambers after 2 to 3 weeks. Electron microscopy showed large numbers of axons present inside the sciatic nerves, irrespective of the presence and number of living Schwann cells. Besides their tendency to fasciculate, axons grew with high preference on Schwann cell membranes and the Schwann cell side of the basal lamina, a situation identical to in vivo regeneration. In contrast to the sciatic nerves, no axons could be found under any condition in the optic nerves. This result points to the existence of extremely poor, non-permissive substrate conditions in the differentiated optic nerves which cannot be overcome by the strong fiber outgrowth-promoting effects of NGF.
\end{abstract}

Neurite outgrowth and regeneration in vivo and in vitro depend on a variety of epigenetic mechanisms. Pure neuronal cultures, particularly in defined media without serum or other rich additives, need specific soluble factors and substrates for optimal survival and neurite production. The best characterized soluble factor is nerve growth factor (NGF), which is necessary for survival and differentiation of sympathetic and subpopulations of sensory neurons (Thoenen and Barde, 1980). Moreover, NGF stimulates the rate of neurite outgrowth and branching frequency and influences the directionality of the growth cone movements (Gundersen and Barret, 1980; Campenot, 1982; Seeley and Greene, 1983). Other factors with different neuronal specificities have been or are being purified from various peripheral organs and from the central nervous system (CNS) (Barde et al., 1982, 1983; Barbin et al., 1984; Berg, 1984). In addition to soluble factors, the culture substrate is essential for neuronal adhesion and influences the number, shape, and growth rate of neurites (Letourneau, 1975; Manthorpe et al., 1983a; Rogers et al., 1983).

Received October 5, 1984; Revised February 27, 1985;

Accepted February 28, 1985

'We thank Mrs. Ch. Müller for excellent technical and photographic assistance, Drs. A. Acheson and D. Edgar for their critical review of the manuscript, and Mrs. E. Eichler and Mrs. H. Macher for their secretarial help.

${ }^{2}$ To whom correspondence should be sent, at: Institute for Brain Research, University of Zurich, August Forel-Str. 7, CH 8029. Zurich, Switzerland
In vivo, the crucial role of the environment through which neurites grow became evident from developmental and regeneration studies. During development, guidance of axons seems to occur in the CNS as well as in the periphery along favored substrate pathways (Katz et al., 1980; Sanes, 1983). In the developing CNS, radial glial cells and glial "bridges" play important roles (Rakic, 1982; Silver et al., 1982). During regeneration in the peripheral nervous system, Schwann cells and basement membranes are very favorable substrates for growing axons (Nathaniel and Pease, 1963; Sanes, 1983). The most impressive effect of the environment on neurite growth and elongation becomes apparent in comparing the regeneration in the CNS and the peripheral nervous system of higher vertebrates. No regeneration of lesioned axonal connections takes place within the differentiated CNS. However, a large variety of central neurons are able to produce axons which elongate over long distances inside a peripheral nerve environment (Ramón y Cajal, 1928; Richardson et al., 1980, 1984; Weinberg and Raine, 1980). Thus, when sciatic nerves are implanted into the spinal cord or the brain, they are invaded by central axons, which, however, stop abruptly at the other end of a "bridge-transplant" when they encounter CNS tissue again (David and Aguayo, 1981). If optic nerves are implanted into sciatic nerves they are almost completely avoided by the regenerating peripheral axons (Aguayo et al., 1978; Weinberg and Spencer, 1979), and the regeneration of dorsal root fibers after a crush of the dorsal root stops at the Schwann cell-CNS interface (Stensaas et al., 1979; Bignami et al., 1984).

The molecular basis of these differences in the microenvironment between the CNS and the peripheral nervous system is largely unknown. Production of specific trophic factors by Schwann cells which induce the regenerating response of the neurons or locally support neurite growth and elongation and the absence of such factors in the adult CNS could be one determinant (Ramón y Cajal, 1928; Kiernan, 1979; Longo et al., 1983). The presence of specific substrates favorable for axonal growth in the periphery and their absence in the CNS is another possibility. In addition, mechanical barriers, e.g., by astrocytic scar tissue, were often discussed as a reason for the lack of CNS regeneration (Ramón y Cajal, 1928; Kiernan, 1979; Reier et al., 1983). All of the in vivo experiments performed so far do not allow one to distinguish between these different hypothetical mechanisms. In particular, it would be of importance to be able to distinguish between the "lack of trophic factor" hypothesis and the presence of a non-permissive substrate for neurite growth in the CNS.

Only in vitro experiments permit the degree of control adequate to define the relative importance of these various influences. In the present study, we used dissociated neurons from neonatal rat sympathetic or sensory ganglia which are grown under optimal culture conditions in the presence of NGF and are capable of regenerating an extensive axonal network. In a chamber culture system these neurons were given the choice to grow into explants of sciatic nerves or optic nerves as "bridges" or "tunnels" to other compartments of the culture dish. By light and electron microscopy, 
axons were observed with high frequency in the sciatic nerve explants, where they were associated with Schwann cells or the Schwann cell side of the basal lamina. Under no condition could axons be seen inside the optic nerve explants.

\section{Material and Methods}

Chamber cultures. A three-compartment Teflon ring as described by Campenot (1979) was covered on one side with silicon high vacuum grease with a syringe. Segments $(3$ to $6 \mathrm{~mm}$ ) of optic or sciatic nerves dissected from adult $(180 \mathrm{gm})$ or 10-day-old Wistar rats were embedded in the grease in such a way that they reached from the central chamber into one of the side chambers (see Fig. 1). In some experiments the nerve explants were frozen (dry ice) and thawed three times before implantation. The rings were then inverted and "glued" onto a $35 \mathrm{~mm}$ tissue culture dish which had been previously coated with collagen.

Dissociated neurons. Superior cervical ganglia or dorsal root ganglia from newborn rats were dissected and cleaned from adhering nerve stumps. They were incubated in $0.1 \%$ trypsin $/ 0.1 \%$ collagenase in $\mathrm{Ca}^{2+} / \mathrm{Mg}^{2+}$-free phosphate-buffered saline at $37^{\circ} \mathrm{C}$ for $45 \mathrm{~min}$, followed by $0.5 \%$ trypsin for 10 min, washed in serum-containing medium, and dissociated by gentle trituration with a Pasteur pipette. The cells were plated into the central slit of the Teflon rings in a volume of $50 \mu \mathrm{l}$ at a density of about $5000 \mathrm{cells} / \mathrm{dish}$; the culture dish was filled up with medium the next day. The cultures were grown in an enriched L15 medium (Mains and Patterson, 1973) with 5\% rat serum, penicillin (100 units $/ \mathrm{ml})$, streptomycin $(100 \mu \mathrm{g} / \mathrm{ml})$, and $50 \mathrm{ng} / \mathrm{ml}$ of $2.5 \mathrm{~S}$ NGF. In some experiments arabinosylcytosine (ara $\mathrm{C} ; 10^{-5} \mathrm{M}$ ) was added for variable times of the culture period (one or two pulses for 2 days at the beginning of the experiment or continuous presence).

The cultures were examined three times a week for the appearance of axons growing out of the nerve explants in the side chambers and for leakage of axons through the silicon grease seal. In 10 experiments a total number of 88 adult sciatic nerve explants, 64 adult optic nerves, and 43 cultures with 10-day-old sciatic and optic nerves were examined. Two experiments with 20 cultures were done with frozen adult nerve explants, with and without ara $\mathrm{C}$.

Electron microscopy. After 2 to 7 weeks cultures were fixed with $2.5 \%$ glutaraldehyde in $0.1 \mathrm{M}$ phosphate buffer $(\mathrm{pH} 7.4)$, washed in phosphate buffer containing $5 \%$ sucrose, and disassembled. The individual nerve explants were postfixed in $1 \% \mathrm{OsO}_{4}$, dehydrated, and embedded in tpon. A total of 30 sciatic and 14 optic nerve explants were studied. Cross-sections of the nerves at various levels were systematically screened for the presence

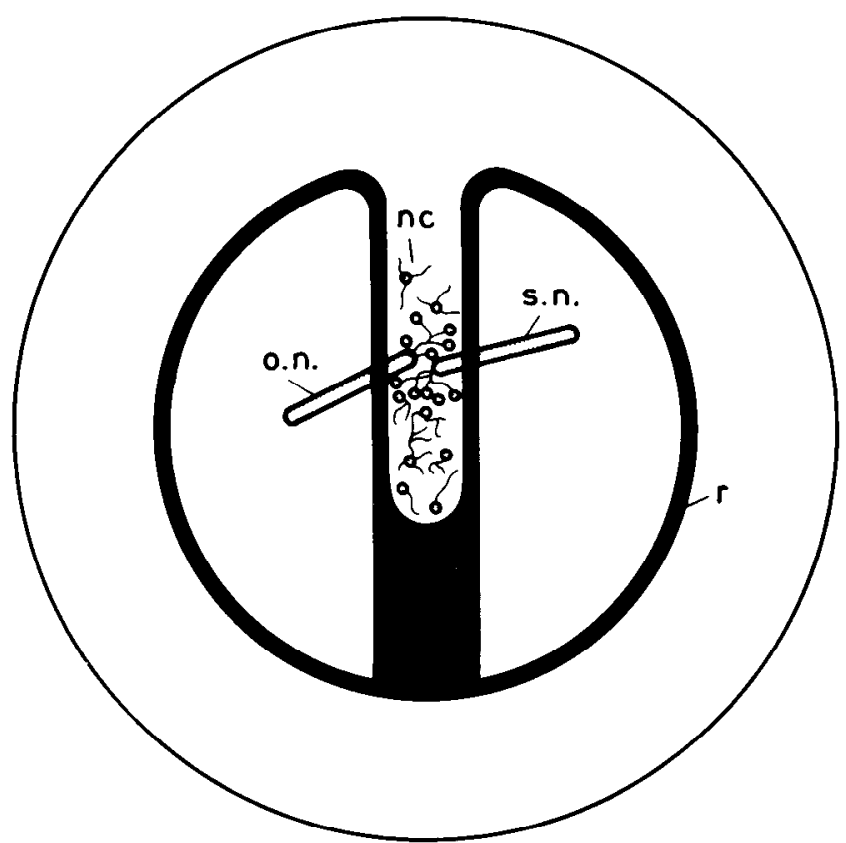

Figure 1. Chamber culture arrangement with Teflon ring ( $r$, optic nerve explant (o.n.), sciatic nerve explant (s.n.), and neurons (nc) in a $35-\mathrm{mm}$ tissue culture dish. of axons in a Zeiss EM 10 electron microscope. For quantitative evaluations all of the axons present in randomly selected fields were photographed.

In vivo experiments. The sciatic nerve of $200 \mathrm{gm}$ female Wistar rats was exposed unilaterally under ether anesthesia and crushed for $40 \mathrm{sec}$ with a watchmaker forceps cooled beforehand in liquid nitrogen. After 4,14 , or 28 days the animals were fixed by perfusion with $2.5 \%$ glutaraldehyde and $1 \%$ formalin in $0.1 \mathrm{M}$ phosphate buffer. Four segments $2 \mathrm{~mm}$ in length were dissected from the sciatic nerve distal to the crush site, postfixed in $1 \%$ $\mathrm{OsO}_{4}$, and embedded in Epon. In some animals the sciatic nerve was transected and the proximal part resected to prevent regencration. The distal stump was studied by electron microscopy (EM) after 4,14 , and 28 days as described above.

\section{Results}

\section{Light microscopic observations}

Sciatic versus optic nerves from adult rats. The majority of the experiments were performed with neurons from the superior cervical ganglion of newborn rats which represent a quite homogeneous population of NGF rosponsive neurons. The presence of NGF $(50$ $\mathrm{ng} / \mathrm{ml}$ ) in the culture medium already led to massive fiber outgrowth after 1 day in culture and to long-term survival of the neurons for more than 2 months. The neurons were plated in the central slit of the culture ring (Fig. 1). In this narrow chamber the axons rapidly contacted the stumps of the sciatic and optic nerves (Fig. 2). After 10 to 20 days in vitro, axons were seen emerging from the distal side of many sciatic nerve explants (about one-fourth of all well placed sciatic nerve explants) (Fig. 3). Often, the number of these penetrating axons was relatively small, but they elongated well on the collagen substrate of the side chamber. In contrast to these light microscopic results many more sciatic nerves containing high numbers of axons were detected by EM (see below).

In the same cultures, sympathetic axons growing out of optic nerve explants into the side chambers could never be observed ( 57 optic nerves examined). However, in some cultures, where leaks occurred in the silicon grease seals, axons were seen to grow along the outside of the optic nerves.

The content of living glial cells in the nerve explants was reduced in some experiments by the addition of cytosine arabinoside to the culture medium. Irrespective of the number of living Schwann and glial cells axons grew through the sciatic nerve explants successfully and did not grow through the optic nerves. This was also true when dead nerve explants were used which had been frozen and thawed three times before implantation into the culture chamber.

Using newborn rat dorsal root ganglion cells, identical results were obtained; axons grew out of sciatic nerve explants in the side chambers but never out of the optic nerve explants.

Sciatic and optic nerve explants from 10-day-old rats. When sciatic and optic nerve explants from 10-day-old rats were used, more non-neuronal cells survived within the explants, and large migration zones formed around the nerves, especially in the side chambers. Again, the regenerating sympathetic or sensory axons frequently grew through the sciatic nerves and over the sciatic nerve migration zones, where association of axons with Schwann cells could be observed. In contrast, 10-day-old optic nerves were also avoided by the axons (43 optic and sciatic nerves examined). Interestingly, when axons invaded the side chambers through leaks in the silicon grease barrier, they also avoided the migration zone around the optic nerve explants (Fig. 4). This finding shows that the non-permissive substrate property of the optic nerves is not restricted to the intact nerve but may also be associated with some type of emigrating non-neuronal cell.

\section{Electron microscopic structure of nerve explants}

In general, many fewer living glial or Schwann cells were present in the cultured adult nerve explants than in denervated nerves in vivo, presumably due to the insufficient diffusion conditions. There- 


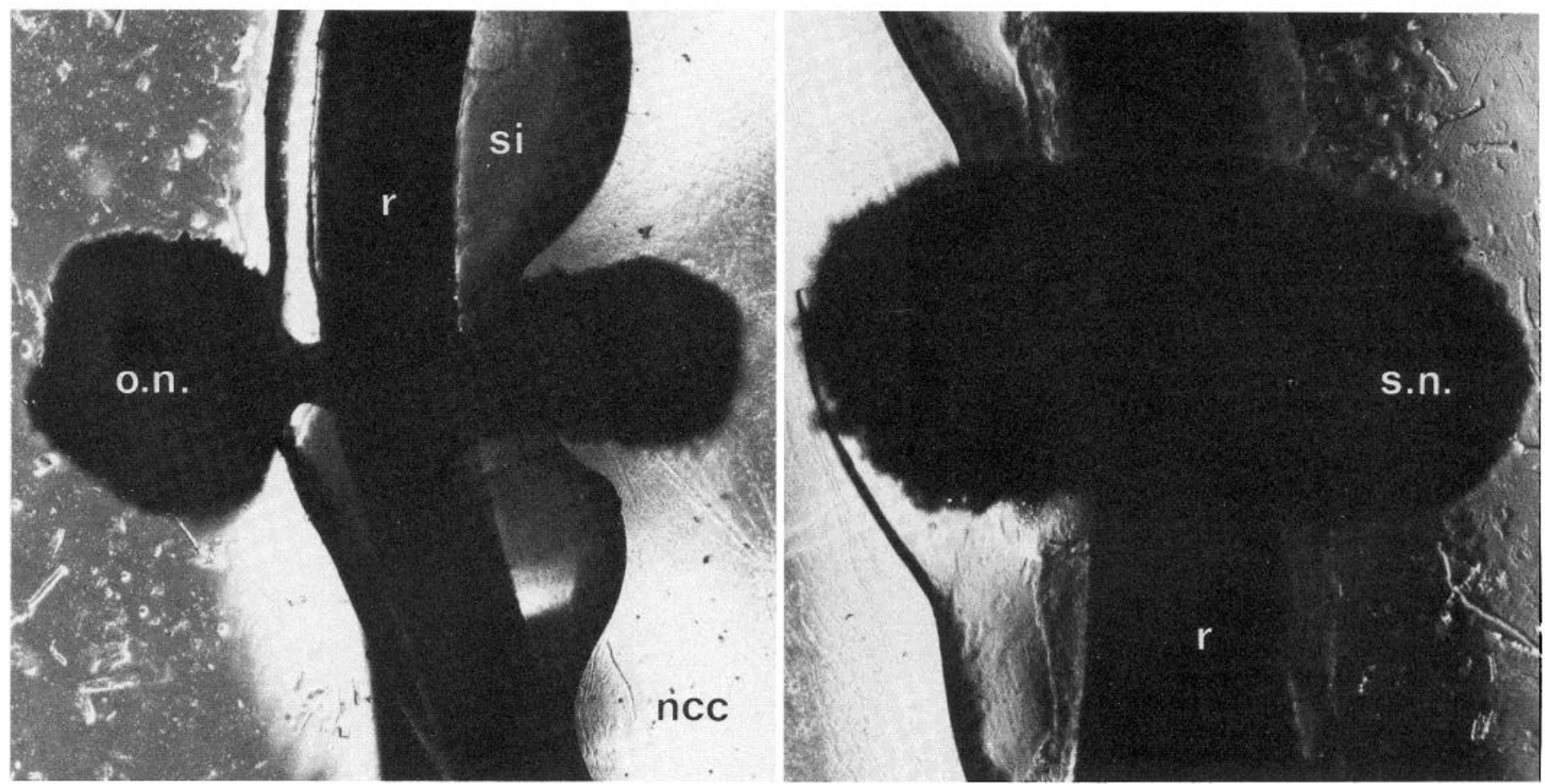

Figure 2. Optic nerve (o.n.) and sciatic nerve (s.n.) explants from adult rats in chamber cultures placed as "bridges" between the nerve cell chamber $(n c c)$ containing sympathetic ganglion cells and the side chambers. $r$, Teflon ring; si, silicon grease. Magnification $\times 32$.

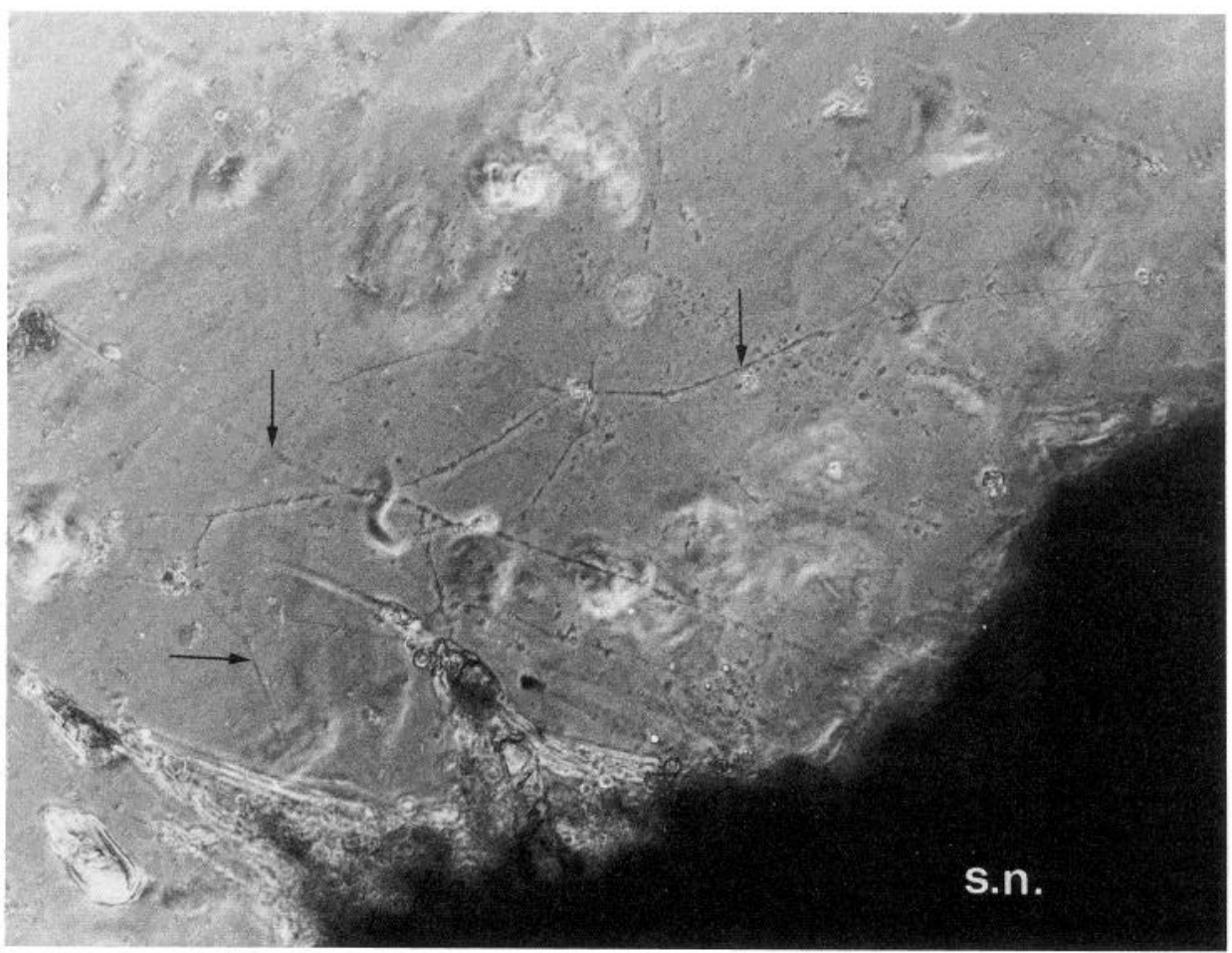

Figure 3. Distal end (side chamber) of an adult sciatic nerve explant (s.n.), 29 days in culture, with axons growing out of the nerve onto the collagen-coated culture dish (arrows). Axons originate from sympathetic neurons in the nerve cell chamber. Magnification $\times 132$.

fore, the phagocytosis of debris and myelin was very incomplete. Empty and partially degraded myelin sheets remained in sciatic as well as optic nerves. They were surrounded by debris of glial cells interspersed with living astrocytes and oligodendrocytes in the optic nerves (Fig. $5 b$ ). Astrocytes were characterized by the presence of the typical intermediate filaments, which were often organized in bundles, and by the frequent occurrence of glycogen and endoplasmic reticulum cisternae with floccular contents (Peters et al., 1976). Oligodendrocytes showed as typical electron microscopic characteristics a high or medium electron-dense cytoplasm, a high amount of heterochromatin, many free polysomes, no intermediate filaments, but often high numbers of microtubules in the processes (Mori and Leblond, 1970). In the sciatic nerve explants, basement membranes covered the myelin-Schwann cell units as they do in vivo, or were found empty, containing only Schwann cell debris (Figs. 5a and 6). Schwann cell processes contained intermediate filaments in addition to microtubules, and their cytoplasm always appeared darker than that of the sympathetic or sensory axons. Spaces between the Schwann cell units were often filled with dense bundles of endoneurial collagen. Phagocytes filled with lipid droplets 


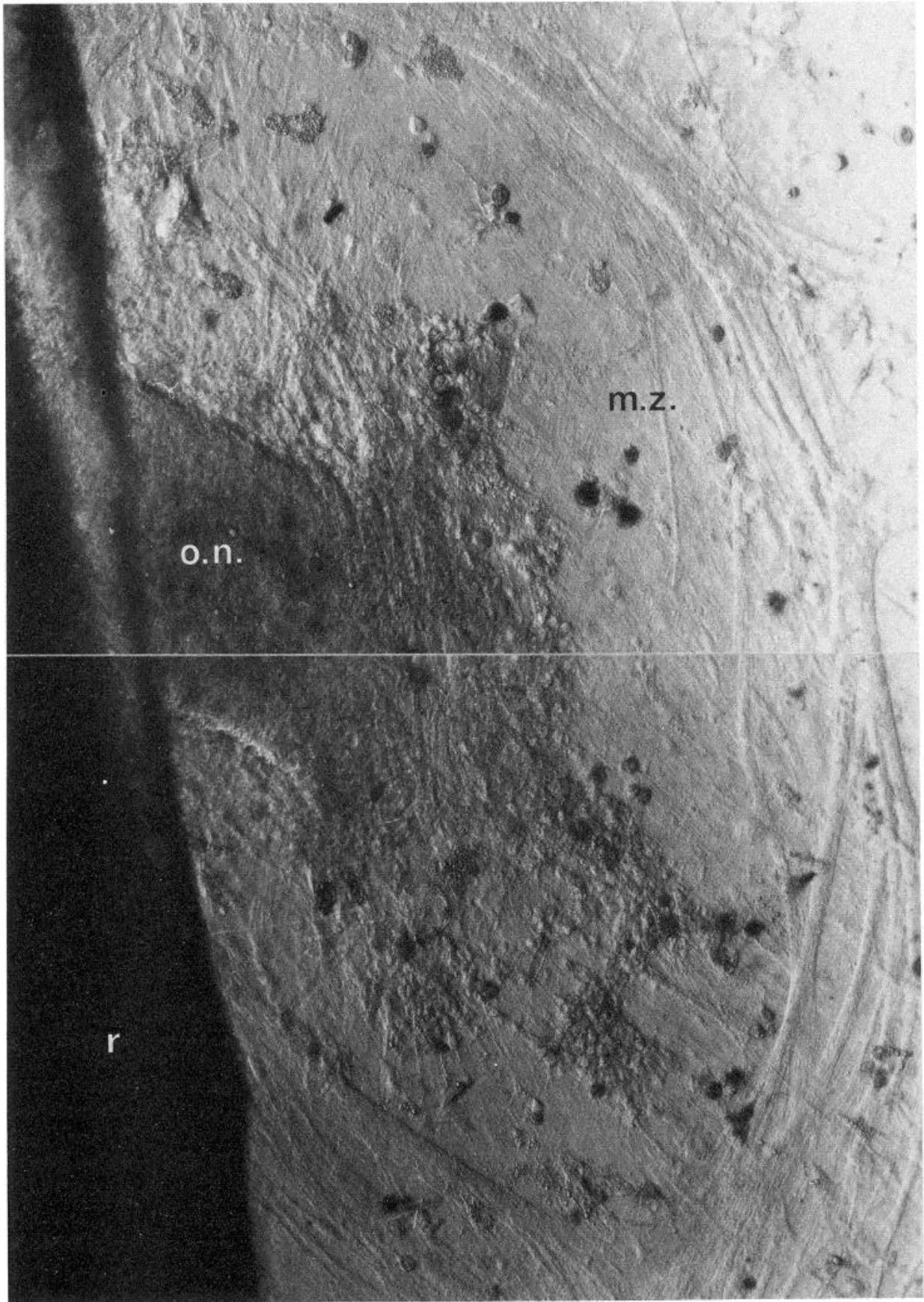

Figure 4. Distal end (side chamber) of an optic nerve explant (o.n.), 22 days in culture, from a 10-day-old rat in a "leaky" culture. Many axons (sympathetic) growing through the leaky silicon grease seal invade the side chamber but avoid the migration zone (m.z.) of the optic nerve glial cells. $r$, Teflon ring. Magnification $\times 128$.

could be observed in both types of nerve explants. The overall density of the tissue and debris varied from explant to explant but was not correlated at all with the presence or absence of axons. Previously frozen, dead optic nerves showed a particularly high degree of structural preservation, especially of cellular membranes.

In the majority of the sciatic nerve explants, sympathetic or sensory axons were present, often in high numbers ( 20 to 400 axons per nerve). Axons could be well distinguished from Schwann cell processes by the lighter cytoplasm, the virtually complete absence of intermediate (neuro-)filaments in these young, regenerating fibers, and the typical wrapping by the Schwann cells. Sympathetic and sensory neurites invaded the sciatic nerves irrespective of the age of the donor animals or the presence or absence of living Schwann cells. Whether age or Schwann cell content had a quantitative influence on the number of axons could not be determined due to the large variations. Frozen adult nerves often contained very large numbers of axons ( 100 to 400 axons per nerve explant) distributed over the entire cross-section of the nerve in spite of the absence of living Schwann cells. In confirmation of the light microscopic observations, no axons were found by electron microscopy in optic nerve explants, living or dead, from adult or 10-day-old animals. In 14 optic nerves screened systematically in the electron microscope only two explants contained a few axons very close to the surface. These axons were in contact with pial collagen and basement membranes, but not with central glial cells. In "leaky" cultures, where neurites grew on the surface of the optic nerves, EM showed that a layer of collagen remained on the surface of the nerves after stripping off the meninges. This finding, that optic nerve surface is favorable for axonal growth, strongly argues against a diffusible inhibitory factor for axonal growth in the optic nerve.

Although typical astrocytes were clearly present in the optic nerve explants (Fig. 5b), astrocytic scars as they appear in vivo after long- 

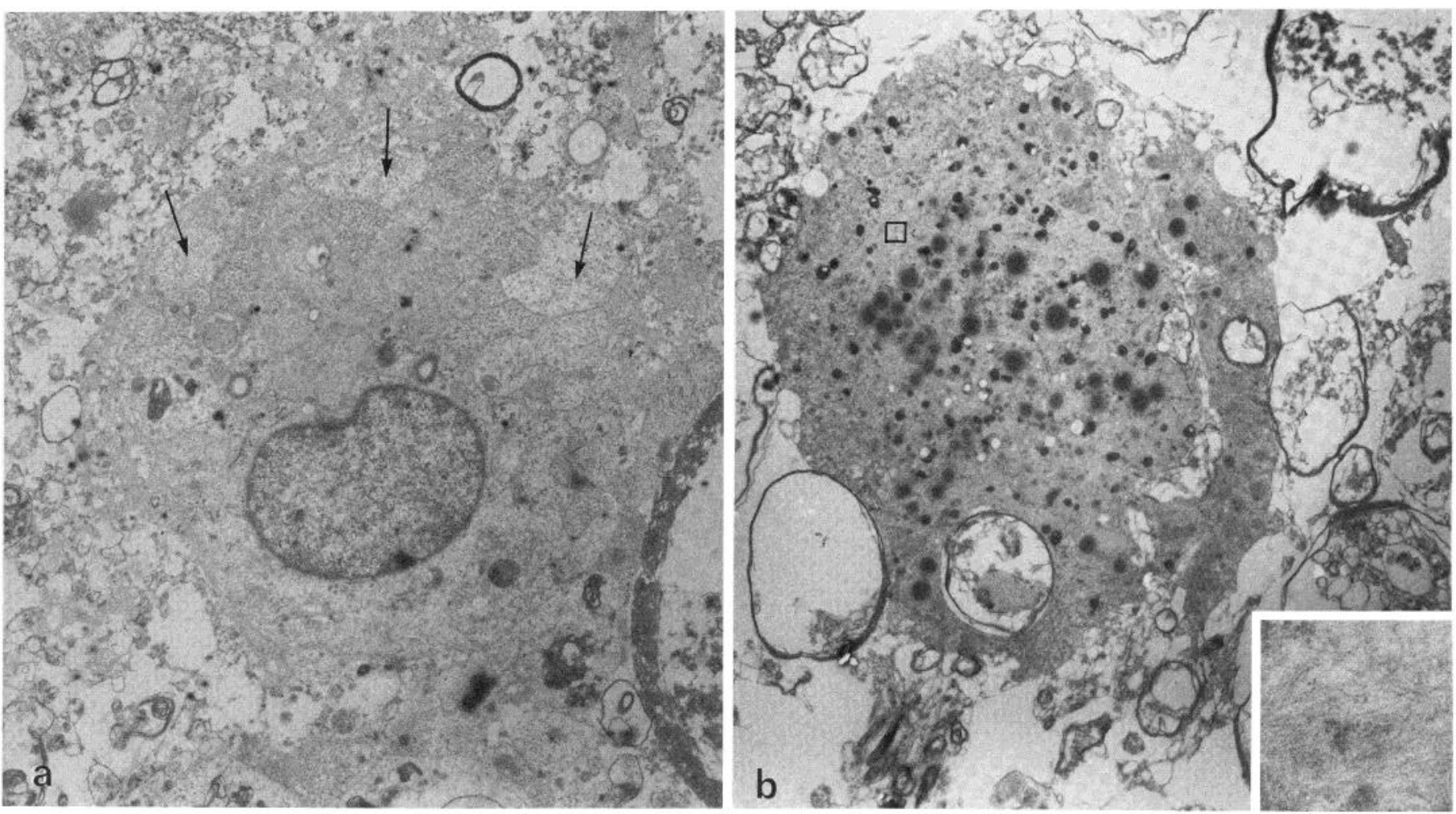

Figure 5. a, Electron micrograph of a Schwann cell surrounded by regenerated axons (arrows) in a sciatic nerve explant (10-day-old rat) co-cultured with sympathetic neurons for 23 days in a chamber culture. Magnification $\times 7,800$. $b$, Astrocyte, characterized by intermediate filaments (inset) in an optic nerve explant (10-day-old rat) co-cultured with sympathetic neurons for 51 days. No axons are present. The square indicates the position of the inset. Magnification: b. $\times 3100$; inset, $\times 25,300$

term denervation could not be observed up to 44 days in culture, probably due to extensive glial cell death. It is, therefore, obvious from these results, that an astrocytic scar cannot be responsible for the total absence of fiber ingrowth into these optic nerve explants under our culture conditions.

\section{Structures associated with axons in the sciatic nerves}

Looking at the structures along which the axons grew inside sciatic nerve explants, it became clear that neighboring axons, the surface of Schwann cells, and the Schwann cell side of the basal lamina were highly preferred substrates. These associations were quantified by recording the neighboring structures on the four sides of each axon. As can be seen from Table I, the most preferred substrate is represented by neighboring axons. This finding corresponds to the well known tendency of axons for fasciculation in vivo and in vitro. If many Schwann cells were present, this axon-axon association was decreased in favor of axon-Schwann cell relationships. Basal lamina was also a preferred substrate. Interestingly, the basal lamina appeared to be highly polarized, since axons were exclusively found on its Schwann cell side. This polarity became most obvious in the dead sciatic nerve explants, where axons were often seen to squeeze themselves between the myelin remnants and the overlying basal lamina (Fig. 6). Endoneurial collagen bundles, myelin, or empty spaces inside the nerve explants were only very rarely used as a substrate by the growing nerve fibers (Table I).

\section{Regenerating sciatic nerves in vivo}

The neighbor relationships of regenerating axons distal to a freezecrush lesion of the sciatic nerves of adult rats in vivo were compared with the present results obtained in chamber cultures. As illustrated in Table II, neighboring axons again were the most preferred substrate at an early stage ( 4 days), followed by Schwann cells and the Schwann cell side of the basal lamina. The axon-axon relations decreased over time $(14,28$ days) when Schwann cells individually wrapped the single axons. Again the localization of axons with regard to the basal lamina was strictly only on its Schwann cell side (Fig. 7). This was particularly obvious in the periphery of the nerves, where, due to the shrinkage after phagocytosis of all of the myelin, large empty spaces were present between the Schwann cell units (Fig. 7).

\section{Discussion}

In the present study, sympathetic or sensory axons were seen to invade and grow through sciatic nerve explants in culture, but never into and through optic nerve explants. This finding corresponds closely to the in vivo situation, where regenerating peripheral and many types of central neurons grow well inside peripheral nerve transplants but do not grow and elongate within the CNS environment (David and Aguayo, 1981; Richardson et al., 1984). Soluble trophic factors, specific substrate conditions, or the mechanical configuration within these two types of tissue could be responsible for this fundamental difference.

In our cultures, neurons were grown in the presence of NGF concentrations which led to optimal, long-term survival and massive fiber outgrowth. The fact that the axons never invaded the optic nerve explants even under these optimal conditions strongly indicates that the lack of a trophic factor cannot be the only essential cause for the failure of axonal growth in this CNS environment.

The fact that not only living but also dead optic nerves were avoided by the axons shows that it is not the production of a soluble inhibitory factor which prevents axon growth inside this CNS tissue. This conclusion is supported by our observation that in cases of leaks between the chambers of the culture ring axons preferably grew along the outside of adult optic nerve explants. Non-permissive substrates, for example, glial membrane components, thus remain an important possibility. In fact, phagocytosis in these frozen optic 

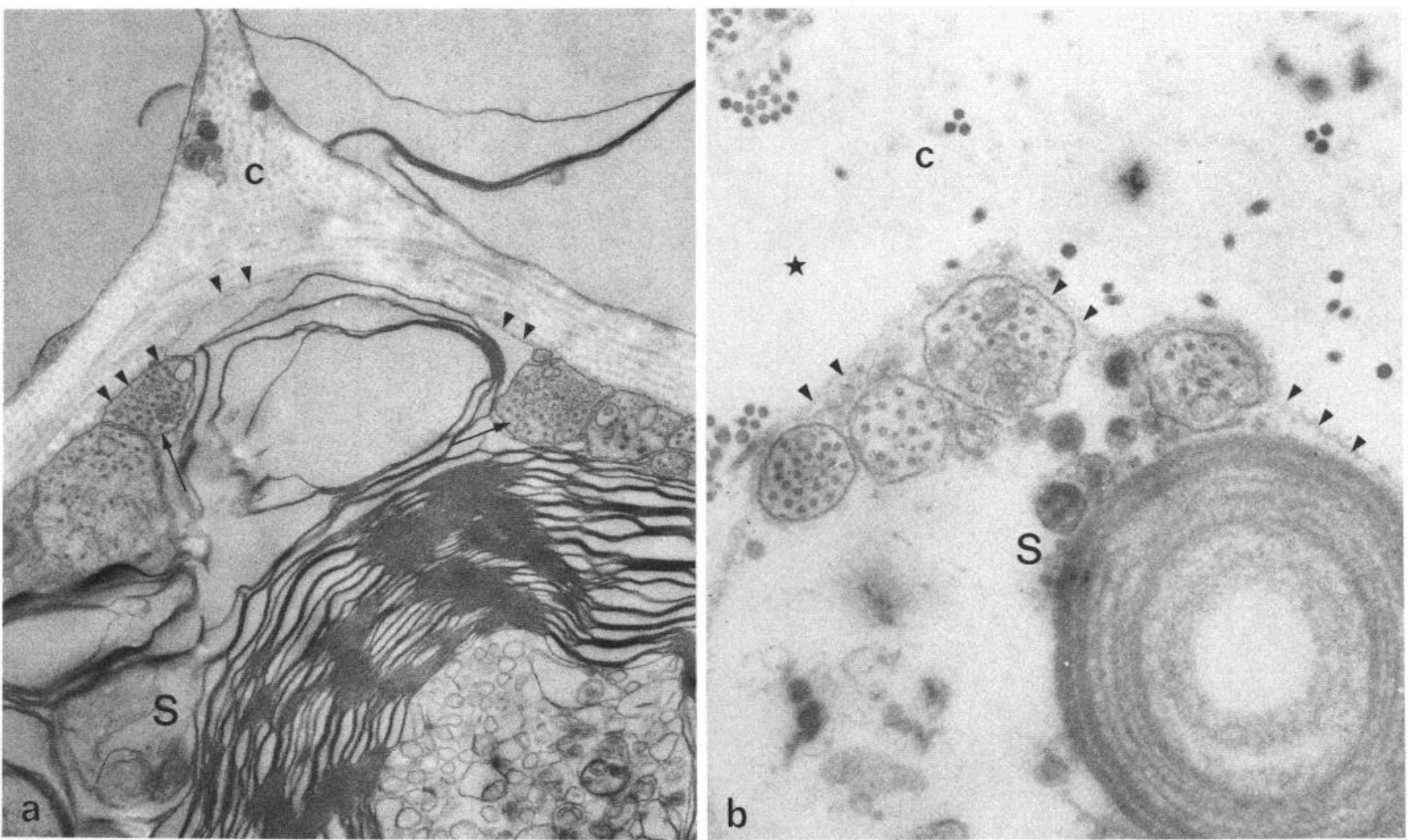

Figure 6. Axons (arrow) regenerating through dead sciatic nerve explants (adult rats) in close apposition to the Schwann cell side of the basal lamina (arrowheads). No axons occur in the large interstitial spaces (star) and on the "fibroblast side" of the basal lamina. $a$, Seventeen days in culture. Magnification $\times 17,000$. b. Thirty-six days in culture. Magnification $\times 40,000$. c, endoneurial collagen; $S$, Schwann cell debris.

TABLE I

Structures in contact with regenerating sympathetic axons in sciatic nerve explants in culture

Randomly sampled axons in EM cross-sections of sciatic nerve explants co-cultred with sympathetic neurons in chamber cultures were analyzed for the structures in contact with a given axon on its four sides. Values represent the means \pm SEM of three to four nerves for each point. Fifty to 150 axons were analyzed for each nerve.

\begin{tabular}{|c|c|c|c|c|c|c|c|c|}
\hline Sciatic Nerves & Other Axons & $\begin{array}{l}\text { Schwann } \\
\text { Cells }\end{array}$ & $\begin{array}{l}\text { Schwann Cell } \\
\text { Basal Lamina }\end{array}$ & $\begin{array}{c}\text { Schwann Cell } \\
\text { Debris; Membranes }\end{array}$ & $\begin{array}{c}\text { Schwann Cell } \\
\text { Debris; Cytoplasm }\end{array}$ & Myelin & Collagen & $\begin{array}{l}\text { Free } \\
\text { Surface }\end{array}$ \\
\hline
\end{tabular}

17 days in vitro

a These living Schwann cells invaded the dead nerves together with the axons. They originate from the superior cervical ganglion.

TABLE ॥

Structures in contact with regenerating sciatic nerve axons in vivo (2 to $4 \mathrm{~mm}$ distal to freeze-crush, unmyelinated axons)

Unmyelinated axons were randomly photographed and the structures contacted by each axon on its four sides were recorded. The values represent the means \pm SEM of three to five nerves for each time point. Fifty to 160 axons were analyzed per nerve.

\begin{tabular}{lccc}
\hline & Other Axons & Schwann Cells & Basal Lamina \\
\hline 4 days after crush & $58 \pm 2.6 \%$ & $25 \pm 3.3 \%$ & $15 \pm 1.2 \%$ \\
14 days after crush & $5 \pm 1.5 \%$ & $80 \pm 3 \%$ & $15 \pm 2 \%$ \\
28 days after crush & $0.3 \pm 0.1 \%$ & $94 \pm 1.5 \%$ & $6 \pm 1.7 \%$ \\
Normal adult nerve & 0 & $95 \%$ & $5 \%$ \\
\hline
\end{tabular}

nerve explants was almost absent and the structural preservation of axonal and glial components and especially their membranes was remarkably good even after 3 weeks in culture.

The importance of living sciatic nerve Schwann cells for the initiation and support of axonal growth was tested by suppressing
Schwann cell proliferation with ara $\mathrm{C}$ or totally eliminating the Schwann cells by prior freezing of the nerve explants. In both cases, sympathetic axons grew well into and through the explants. Thus, the continuous production of some "factors" by denervated living Schwann cells is not an absolute prerequisite, at least in the presence of added exogenous NGF. Under normal in vivo conditions, sciatic nerve Schwann cells do not contain significant amounts of NGF (Korsching and Thoenen, 1983). However, an increased NGF (and other factor) production in response to denervation, which could substitute for the interrupted supply of NGF by retrograde transport from the target organ (Schwab and Thoenen, 1983), has to be considered (Longo et al., 1983, Richardson and Ebendal, 1982; S. Korsching and $\mathrm{H}$. Thoenen, unpublished observations).

The overall density of the tissue was variable but comparable in living optic and sciatic nerve explants. There was no correlation between tissue density and axon ingrowth in the sciatic nerves; axons were seen to also penetrate very dense regions of the explants. This finding corresponds well to observations in the optic 

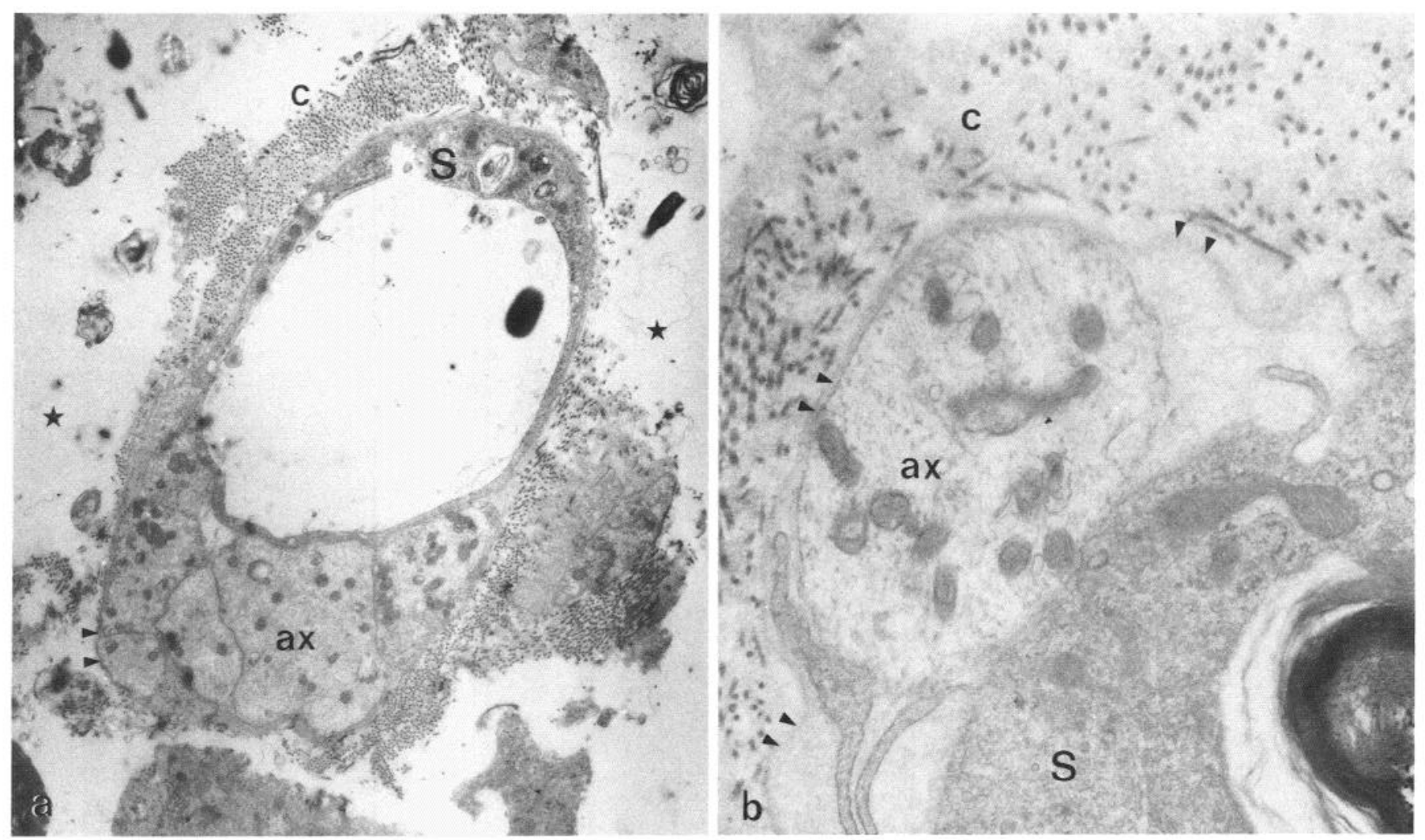

Figure 7. Regenerating axons (ax) in adult rat sciatic nerves in vivo distal to a freeze-crush placed 4 days earlier. Axons grow exclusively in contact with the Schwann cell $(S)$ and its basal lamina (arrowheads) in spite of the large endoneurial spaces in the shrunken nerve trunk (stars). $C$, endoneurial collagen. Magnification: $a, \times 5,000 ; b, \times 21,800$.

nerve of Xenopus, where it was found that even dense astrocytic scar tissue did not inhibit optic nerve regeneration (Reier, 1979). Astrocytic scar tissue as it is seen after long-term denervation in vivo in rat optic nerves or at the site of a CNS lesion did not develop under the present conditions. Thus, axons in culture even avoided the optic nerves in the absence of such mechanical barriers.

The major differences between optic and sciatic nerves are the different types of glial cells and the exclusive presence of basement membranes in the peripheral nerves. In fact, the preferential localization of the axons within our sciatic nerve explants points to an important role of specific substrates. Besides the well known tendency for fasciculation, which could be mediated by N-CAM (Edelman, 1984), axons grew mostly in contact with the surface of living Schwann cells and the Schwann cell side of the basal lamina. This association with basal laminae was particularly striking in the dead sciatic nerve explants, where axons often squeezed themselves between the myelin, the Schwann cell debris, and the overlaying basal lamina. In contrast, axons were absent from the often large, empty spaces between the original nerve-Schwann cell units. This high preference of axons for Schwann cells and their basal laminae is well known for in vivo regeneration and has recently also been shown in reimplanted frozen sciatic nerve segments (Nathaniel and Pease, 1963; Ide et al., 1983).

That the basal lamina is polarized is not astonishing in view of its production by Schwann cells from one side and fibroblasts from the other. In fact, in rat sciatic nerves an uneven distribution of anionic sites was recently shown using cationic ferritin and ruthenium red as markers (Yokota et al., 1983). Obviously, for comparison with the CNS, it would be of importance to know the biochemical nature of the component(s) of Schwann cell surfaces and basement membranes that make them such favorable substrates for axonal growth. Major constituents of basement membranes are collagen type IV, laminin, and fibronectin. All of these constituents seem to be good substrates for various types of dissociated neurons in culture (Rogers et al., 1983). Particularly outstanding is laminin, which represents an excellent culture substrate for a variety of peripheral and central neurons (Baron-van Evercooren et al., 1982; Manthorpe et al., 1983a; Rogers et al., 1983). Laminin is present in the endoneurial basement membranes of sciatic nerves and seems to come mainly from the Schwann cells, which also contain laminin on their surfaces in culture (Cornbrooks et al., 1983; Palm and Furcht, 1983).

Except for the basement membranes formed by astrocytic endfeet around blood vessels and on the CNS surface, no basement membranes are present in brain or spinal cord, and major basement membrane constituents like laminin, collagen IV, or fibronectin have not been detected in normal adult brain parenchyma (Liesi et al., 1984; Schachner et al., 1978). Astrocytes in culture and reactive astrocytes at the site of CNS lesions start to produce and secrete laminin and can form basement membranes (Liesi et al., 1983, 1984; Bernstein et al., 1985). In the case of our optic nerve explants, immunofluorescence experiments with an antiserum against laminin showed a pronounced staining of astrocytes ( 28 days in culture), although ectopic basal laminae were rarely observed by EM (M. E. Schwab, unpublished observations). Nevertheless, this laminin production in the nerve explants or at lesion sites in vivo does not seem to be capable of or sufficient to support axonal regeneration. Therefore, besides the possible lack of substrates favoring neurite growth in the differentiated CNS, inhibitory substrate molecules should be considered. In vitro, dissociated astrocytes are favorable substrates for a variety of neurons (Lindsay et al., 1982; Noble et al., $1984 ;$ M. E. Schwab, unpublished observations). In contrast, the migration zones in the side chamber around the optic nerve explants from 10-day-old rats in our cultures were seen to be avoided by growing nerve fibers. Among the non-neuronal cells found in these 
migration areas are very typical highly branched cells positive for the 04-antigen, a marker for oligodendrocytes, immature oligodendrocytes, and Schwann cells (Schachner et al., 1981; Sommer and Schachner, 1981). The same cells, which are also positive for galactocerebroside, are strongly avoided by the growing neurites if co-cultured with dissociated sympathetic or dorsal root ganglion cells or retina cells (M. E. Schwab, manuscript in preparation). These results suggest that these glial cells possess surface characteristics or produce local substrate modifications which do not allow axonal growth even under optimal trophic factor conditions. The present results do not rule out that trophic factors are important and can be a prerequisite for successful regeneration for peripheral and CNS neurons. Trophic factors are present in adult brain (Barde et al., 1982) and are also produced by cultured astrocytes (Banker, 1980; Lindsay et al., 1982) or in regions of CNS lesions (Nieto-Sampedro et al., 1982; Manthrope et al., 1983b). Nevertheless, the strong effect of NGF in our cultures could not overcome the inhibition exerted by the substrate inside the optic nerve explants.

\section{References}

Aguayo, A. J., R. Dickson, J. Trecarten, and M. Attiwell (1978) Ensheathment and myelination of regenerating PNS fibers by transplanted optic nerve glia. Neurosci. Lett. 9: 97-104.

Banker, G. A. (1980) Trophic interactions between astroglial cells and hippocampal neurons in culture. Science 209: 809-810.

Barbin, G., M. Manthorpe, and S. Varon (1984) Purification of the chick eye ciliary neuronotrophic factor. J. Neurochem. 43: 1468-1478.

Barde, Y. A., D. Edgar, and $H$. Thoenen (1982) Purification of a new neurotrophic factor from mammalian brain. EMBO J. 1: 549-553.

Barde, Y. A., D. Edgar, and H. Thoenen (1983) New neurotrophic factors. Annu. Rev. Physiol. 45: 601-612.

Baron-van Evercooren, A., H. K. Kleinman, S. Ohno, D. Marangos, J. P. Schwartz, and M. E. Dubois-Dalcq (1982) Nerve growth factor, laminin, and fibronectin promote neurite growth in human fetal sensory ganglia cultures. J. Neurosci. Res. 8: 179-193.

Berg, D. K. (1984) New neuronal growth factors. Annu. Rev. Neurosci. 7: $149-170$.

Bernstein, J. J., R. Getz, M. Jefferson, and M. Kelemen (1985) Astrocytes secrete basal lamina after hemisection of rat spinal cord. Brain Res. 327: 735-747.

Bignami, A., N. M. Chi, and D. Dahl (1984) Regenerating dorsal roots and the nerve entry zone: An immunofluorescence study with neurofilament and laminin antisera. Exp. Neurol. 85: 426-436.

Campenot, R. B. (1979) Independent control of the local environment of somas and neurites. Methods Enzymol. 58: 302-307.

Campenot, R. B. (1982) Development of sympathetic neurons in compartmentalized cultures. I. Local control of neurite growth by nerve growth Iaclur. Dev. Biul. 93: 1-12

Cornbrooks, C. J., D. J. Carey, J. A. McDonald, R. Timpl, and R. P. Bunge (1983) in vivo and in vitro observations on laminin production by Schwann cells. Proc. Natl. Acad. Sci. U. S. A. 80: 3850-3854.

David, S., and A. J. Aguayo (1981) Axonal elongation into peripheral nervous system "bridges" after central nervous system injury in adult rats. Science 214: 931-933.

Edelman, G. M. (1984) Modulation of cell adhesion during induction, histogenesis, and perinatal development of the nervous system. Annu. Rev. Neurosci. 7: 339-377.

Gundersen, R. W., and J. N. Barrett (1980) Characterization of the turning response of dorsal root neurites toward nerve growth factor. J. Cell Biol. 87: 546-554.

Ide, C., K. Tohyama, R. Yokota, T. Nitatori, and S. Onodera (1983) Schwann cell basal lamina and nerve regeneration. Brain Res. 288: 61-75.

Katz, M., R. J. Lasek, and H. J. W. Nauta (1980) Ontogeny of substrate pathways and the origin of the neural circuit pattern. Neuroscience 5: 821833.

Kiernan, J. A. (1979) Hypotheses concerned with axonal regeneration in the mammalian nervous system. Biol. Rev. 54: 155-197.

Korsching, S., and $H$. Thoenen (1983) Quantitative demonstration of the retrograde axonal transport of endogenous nerve growth factor. Neurosci. Lett 39: 1-4.
Letourneau, P. C. (1975) Possible roles for cell-to-substratum adhesion in neuronal morphogenesis. Dev. Biol. 44: 77-91.

Liesi, P., D. Dahl, and A. Vaheri (1983) Laminin is produced by early rat astrocytes in primary culture. J. Cell Biol. 92: 920-924.

Liesi, P., S. Kaakkola, D. Dahl, and A. Vaheri (1984) Laminin is induced in astrocytes of adult brain by injury. EMBO J. 3: 683-686.

Lindsay, R. M., P. C. Barber, M. R. C. Sherwood, J. Zimmer, and G. Raisman (1982) Astrocyte cultures from adult rat brain. Derivation, characterization and neurotrophic properties of pure astroglial cells from corpus callosum. Brain Res. 243: 329-343.

Longo, F. M., M. Manthorpe, S. D. Shaper, G. Lundborg, and S. Varon (1983) Neuronotrophic activities accumulate in vivo within silicone nerve regeneration chambers. Brain Res. 261: 109-117.

Mains, R. E., and P. H. Patterson (1973) Primary cultures of dissociated sympathetic neurons. J. Cell Biol. 59: 329-345.

Manthorpe, M., E. Engvall, E. Ruoslakti, F. M. Longo, G. E. Davis, and S. Varon (1983a) Laminin promotes neuritic regeneration from cultured peripheral and central neurons. J. Cell Biol. 97: 1882-1890.

Manthorpe, M., M. Nieto-Sampedro, S. D. Skaper, E. R. Lewis, G. Barbin, F. M. Longo, C. W. Cotman, and S. Varon (1983b) Neuronotrophic activity in brain wounds of the developing rat. Correlation with implant survival in the wound cavity. Brain Res. 267: 47-56.

Mori, S., and C. P. I eblond (1970) Electron microscopic identification of three classes of oligodendrocytes and a preliminary study of their proliferative activity in the corpus callosum of young rats. J. Comp. Neurol. 139: $1-30$.

Nathaniel, E. J. H., and D. C. Pease (1963) Regenerative changes in rat dorsal roots following Wallerian degeneration. J. Ultrastruct. Res. 9: 533549 .

Nieto-Sampedro, M., E. R. Lewis, C. W. Cotman, M. Manthorpe, S. D. Skaper, G. Barbin, F. M. Longo, and S. Varon (1982) Brain injury causes a timedependent increase in neuronotrophic activity at the lesion site. Science 217: 860-861.

Noble, M., J. Fok-Seang, and J. Cohen (1984) Glid are a unique substrate for the in vitro growth of central nervous system neurons. J. Neurosci. 4: 1892-1903.

Palm, S. L., and L. T. Furcht (1983) Production of laminin and fibronectin by Schwannoma cells: Cell-protein interactions in vitro and protein localization in peripheral nerve in vivo. J. Cell Biol. 96: 1218-1226.

Peters, A., S. L. Palay, and H. F. Webster (1976) The Fine Structure of the Nervous System, Ed. 2, Harper and Row, New York.

Rakic, P. (1982) The role of neuronlal-glial cell interaction during brain development. in Neuronal-Glial Cell Interrelationships, S. A. Sears, ed., pp. 25-78, Springer-Verlag, New York.

Ramón y Cajal, S. (1928) Degeneration and Regeneration of the Nervous System, English translation and reprint (1959). Hafner Press, New York.

Reier, P. J. (1979) Penetration of grafted astrocytic scars by regenerating optic nerve axons in Xenopus tadpoles. Brain Res. 164: 61-68.

Reier, P. J., L. J. Stensaas, and L. Guth (1983) The astrocytic scar as an irmpediment to regeneration in the central nervous system. In Spinal Cord Reconstruction, C. C. Kao, R. P. Bunge, and P. J. Reier, eds., pp. 163195, Raven Press, New York.

Richardson, P. M., and T. Ebendal (1982) Nerve growth activities in rat peripheral nerve. Brain Res. 246: 57-64.

Richardson, P. M., U. M. McGuinness, and A. J. Aguayo (1980) Axons from CNS neurons regenerate into PNS grafts. Nature 284: 264-265.

Richardson, P. M., V. M. K. Issa, and A. J. Aguayo (1984) Regeneration of long spinal axons in the rat. J. Neurocytol. 13: 165-182.

Rogers, S. L., P. C. Letourneau, S. L. Palm, J. McCarthy, and L. T. Furcht (1983) Neurite extension by peripheral and central nervous system neurons in response to substratum-bound fibronectin and laminin. Dev. Biol. 98: 212-220.

Sanes, J. R. (1983) Roles of extracellular matrix in neural development. Annu Rev. Physiol. 45: 581-600.

Schachner, M., G. Schoonmaker, and R. O. Hynes (1978) Cellular and subcellular localization of LETS protein in the nervous system. Brain Res. 158: $149-158$.

Schachner, M., S. K. Kim, and R. Zehnle (1981) Developmental expression in central and peripheral nervous system of oligodendrocyte cell surface antigens ( $\mathrm{O}$ antigens) recognized by monoclonal antibodies. Dev. Biol. 83: 328-338.

Schwab, M. E., and H. Thoenen (1983) Retrograde axonal transport. In 
Handbook of Neurochemistry, A. Lajtha, ed., Vol. 5, pp. 381-404, Plenum Press, New York.

Seeley, P. J., and L. A. Greene (1983) Short-latency local actions of nerve growth factor at the growth cone. Proc. Natl. Acad. Sci. U. S. A. 80: 2789 2793.

Silver, J., S. E. Lorenz, D. Wahlstein, and J. Coughlin (1982) Axonal guidance during development of the great cerebral commissures: Descriptive and experimental studies, in vivo, on the role of preformed glial pathways. J. Comp. Neurol. 210: 10-29.

Sommer, I., and M. Schachner (1981) Monoclonal antibodies (01 to 04) to oligodendrocyte cell surfaces: An immunocytological study in the central nervous system. Dev. Biol. 83: 311327.
Stensaas, L. J., P. R. Burgess, and K. W. Horch (1979) Regenerating dorsal root axons are blocked by spinal cord astrocytes. Soc. Neurosci. Abstr. 5: 684 .

Thoenen, H., and Y. A. Barde (1980) Physiology of nerve growth factor. Physiol. Rev. 60: 1284-1335.

Weinberg, E. L., and P. S. Spencer (1979) Studies on the control of myelinogenesis. 3 . Signalling of oligodendrocyte myelination by regenerating peripheral axons. Brain Res. 162: 273-279.

Weinberg, E. L., and C. S. Raine (1980) Reinnervation of peripheral nerve segments implanted into the rat central nervous system. Brain Res. 198 : $1-11$.

Yokota, R., K. Tohyama, and C. Ide (1983) Distribution of anionic sites on the basal lamina of Schwann cells. Neurosci. Lett. 41: 49-54. 